\title{
Alterações neuroanatômicas do encéfalo na malformação Arnold-Chiari II
}

\author{
Encephalic Neuroanatomical alterations in Arnold-Chiari II malformation
}

Alteraciones neuroanatómicas del encéfalo en la malformación Arnold-Chiari II

Marcelo José da Silva de Magalhães ${ }^{1 *}$, Brunno Pedreira Montenegro Pimenta ${ }^{2}$, Heitor Oliveira Gomes $^{2}$, José Valci Fernandes Neto², Rônney Vasconcelos de Oliveira², Sophia Santos Novais².

\section{RESUMO}

Objetivo: Descrever as alterações neuroanatômicas do SNC encontradas nos exames de neuroimagem nos pacientes com malformação de Chiari II. Métodos: Trata-se de uma revisão descritiva baseada em artigos provenientes da base de dados BVS, Scielo e PubMed. Foram utilizados artigos compreendidos em um período de 21 anos (entre 1997 e 2016). Os descritores utilizados foram "Chiari II", "fetal surgery", "hydrocephalus", "intrauterine surgery", "myelomeningocele", "prenatal", "symptomatic Chiari II", "spina bifida" e "spinal dysraphisms". Resultados: As malformações de Chiari II encontram-se inseridas no espectro de anormalidades congênitas do sistema nervoso central (SNC). É caracterizada por uma deformidade complexa da fossa posterior associada quase sempre a uma malformação da coluna vertebral. As alterações encefálicas mais sugestivas dessa patologia são anormalidades cerebelares; deslocamento caudal da ponte, IV ventrículo e bulbo; torção medular; IV ventrículo em formato anormal e hipoplasia da tenda do cerebelo e do teto do mesencéfalo. O diagnóstico pode ser realizado através da ultrassonografia, tomografia computadorizada e ressonância magnética. Considerações finais: A Malformação de Chiari II é uma doença que apresenta quadro clínico e alterações radiológicas complexas e extensas. Mais estudos que considerem as alterações morfológicas são necessários.

Palavras-chave: Malformação de Arnold-Chiari, Meningomielocele, Disrafismo espinal, Hidrocefalia.

\begin{abstract}
Objective: To describe the CNS neuroanatomical alterations found in neuroimaging studies in patients with Chiari II malformation. Methods: This is a descriptive review based on articles from the BVS, Scielo and PubMed database. Articles were used over a period of 21 years (between 1997 and 2016). The therms used were "Chiari II", "fetal surgery", "hydrocephalus", "intrauterine surgery", "myelomeningocele", "prenatal", "symptomatic Chiari II", "spina bifida" and "spinal dysraphisms". Results: Chiari II malformations are inserted into the spectrum of congenital abnormalities of the central nervous system (CNS). It is characterized by a complex deformity of the posterior fossa almost always associated with a malformation of the vertebral column. The most suggestive encephalic changes of this pathology are cerebellar abnormalities; caudal displacement of the bridge, IV ventricle and bulb; medullar torsion; IV abnormally shaped ventricle and hypoplasia of the cerebellum tent and midbrain roof the diagnosis can be made through ultrasonography, computed tomography and magnetic resonance imaging. Final considerations: Chiari II malformation is a disease that presents a clinical picture and complex and extensive radiological changes. More studies that consider morphological changes are needed.
\end{abstract}

Key words: Malformation of Arnold-Chiari, Meningomyelocele, Spinal Dysraphism, Hydrocephalus.

${ }^{1}$ Centro Universitário FipMoc, Montes Claros-MG. *E-mail: marcelo7779@yahoo.com.br

2Departamento de Medicina das Faculdades Unidas do Norte de Minas (FUNORTE).

SUBMETIDO EM: 3/2019 


\section{RESUMEN}

Objetivo: Describir las alteraciones neuroanatómicas del SNC encontradas en los exámenes de neuroimagen en los pacientes con malformación de Chiari II. Metodología: Se trata de una revisión descriptiva basada en artículos provenientes de la base de datos BVS, Scielo y PubMed. Se utilizaron artículos comprendidos en un período de 21 años (entre 1997 y 2016). Los descriptores utilizados fueron "Chiari II", "fetal surgery", "hydrocephalus", "intrauterine surgery", "myelomeningocele", "prenatal", "sintomatic Chiari II", "spina bifida" y "spinal dysraphisms". Resultados: Las malformaciones de Chiari II se encuentran insertadas en el espectro de anormalidades congénitas del sistema nervioso central (SNC). Se caracteriza por una deformidad compleja de la fosa posterior asociada casi siempre a una malformación de la columna vertebral. Las alteraciones encefálicas más sugestivas de esta patología son anormalidades cerebelares; desplazamiento caudal del puente, IV ventrículo y bulbo; torsión medular; IV ventrículo en forma anormal e hipoplasia de la tienda del cerebelo y del techo del mesencéfalo El diagnóstico puede ser realizado a través de la ultrasonografía, tomografía computarizada y resonancia magnética. Consideraciones finales: La malformación de Chiari II es una enfermedad que presenta cuadro clínico y alteraciones radiológicas complejas y extensas. Más estudios que consideren los cambios morfológicos son necesarios.

Palabras-clave: Malformación de Arnold-Chiari, Meningomyelocele, SpinalDysraphism, Hydrocephalus.

\section{INTRODUÇÃO}

As malformações de Chiari II (MCII) encontram-se inseridas no espectro de anormalidades congênitas do sistema nervoso central. É caracterizada por uma deformidade complexa da fossa posterior associada quase sempre a uma mielodisplasia - a mielomeningocele resulta de um defeito do fechamento do tubo neural ao nível lombossacral. Além da herniação cerebelar e disrafismo espinhal, encontramos alterações no tronco encefálico, no sistema ventricular, no mesoderma paraxial do crânio e nos hemisférios cerebrais (OSBORN et al., 2014).

Inicialmente, foi descrita ao final do século XIX por Hans Chiari no conjunto de herniações cerebelares, adquirindo o epônimo clássico de Arnold-chiari em 1907 por Schwalbe e Gredig, discípulos de Arnold ao acrescentar à doença o quadro de mielomeningocele. No entanto, considera-se limitada a sua participação, sendo restrita a um caso apenas. (PENFIELD e COBURN, 1938)

A prevalência geral de Chiari Il descrita nos Estados Unidos é de 0,44 em 1.000 nascidos vivos, no entanto tem-se reduzido devido à profilaxia com ácido fólico diário na dose de $4 \mathrm{mg}$ (reduz o risco em aproximadamente 70\%). No Brasil, estudos feitos pela UNICAMP no Programa de Genética Perinatal no período de 1982 a 2001 indicou um resultado ainda maior, com 2,28 para cada 1000 nascimentos (SBRAGIA et al., 2004).

A malformação de Chiari do tipo II está relacionada a uma elevada taxa de mortalidade. O comprometimento do tronco cerebral e dos nervos cranianos pode provocar pneumonias de repetição relacionadas à disfagia e à perda dos reflexos orofaríngeo e traqueobrônquico. Crises de apneia também são responsáveis por grande parte das mortes e ocorrem devido à paralisia dos abdutores das cordas vocais e obstrução mecânica pela aspiração de leite materno. Além disso, os déficits neurológicos podem cursar com hidrocefalia, atrasos no desenvolvimento neuropsicomotor, incontinência urinária, déficits sensório-motores, paraparesia e tetraparesia, representando elevada morbidade dessa malformação congênita, com grande impacto social na vida dos pais e cuidadores desses pacientes (GRIEBEL et al., 1991; MCLONE, 1992).

Dentre as diferentes modalidades de tratamento cirúrgico, a derivação ventrículo peritoneal (DVP) está entre as opções mais utilizadas para a correção da hidrocefalia, muito frequentemente associada a esta malformação. Nota-se que existem diferentes modalidades de derivação além da DVP. Esta cirurgia apresenta índices de complicações consideráveis, tais como infecção, obstrução do sistema e falhas do sistema mecânico do dispositivo. (PEREIRA et al., 2007). 
Muitas vezes, devido as alterações neuroanatômicas da fossa posterior, há necessidade de correção cirúrgica dessa deformidade através de craniectomia dessa parte do crânio. Essa modalidade de tratamento cirúrgico pode estar associada ou não à laminectomia da coluna cervical. (STEVENSON, 2004).

Atualmente, tem-se realizado também o tratamento intra-útero dessa doença da malformação da mielomeningocele, trazendo resultados animadores quando comparados as outras modalidades disponíveis de tratamento. (BEVILACQUA NS, PEDREIRA DAL, 2015)

Este trabalho tem como objetivo revisar as alterações neuroanatômicas encontradas na malformação de Chiari II, levando em conta a importância dessas alterações na fisiopatologia da doença e no tratamento cirúrgico.

\section{MÉTODO}

Trata-se de uma revisão descritiva baseada em artigos da base de dados BVS, Scielo e PubMed. Foram utilizados artigos compreendidos em um período de 21 anos (entre 1997 e 2016). Os descritores utilizados foram "Chiari II", "fetal surgery", "hydrocephalus", "intrauterine surgery", "myelomeningocele", "prenatal", "symptomatic Chiari II", "spina bifida" e "spinal dysraphisms".

Foram incluídos somente artigos em língua inglesa ou portuguesa. Também foram adicionados artigos de opinião, revisão, relatos de casos e estudos populacionais. Foram utilizadas as seguintes referências bibliográficas: Osborn Brain (2013) e The spina bifida: Managment and outcome (2008). Artigos citados pelos artigos selecionados, mas anteriores a 1997, foram adicionados quando muito relevantes do ponto de vista histórico. Foram excluídos artigos científicos que apresentavam somente aspectos éticos e cirúrgicos.

\section{RESULTADOS/DISCUSSÃO}

\section{Epidemiologia}

A prevalência geral da Malformação de Chiari tipo II é de 0,44 em 1000 nascidos vivos, entretanto tem-se reduzido com a terapia profilática materna com ácido fólico. A dose de $4 \mathrm{mg} /$ dia reduz pelo menos $70 \%$ do risco de MCII (OSBORN et al., 2014). Cai C e Oakes WJ (1997) descreveram malformações de Chiari em 1/3 dos afetados com mielomeningocele. Waters et al. (1998) narram a incidência de mielomeningocele em 0,5 crianças a cada 1000 nascidas vivas e associam a malformação de Chiari tipo II a esta anomalia. McLone (1983) comenta que $32 \%$ dos pacientes com mielomeningocele possam apresentar concomitantemente a Malformação de Chiari tipo II.

\section{Fisiopatologia}

Penfield e Cuborn (1938) hipotetiza que a MCll seria secundária a um processo de tracionamento realizado pelo ancoramento da medula ao nível mielomeningocele. Por outro lado, Griebelet al. (1991), não concorda que esse mecanismo de tração como justificativa para as demais alterações neuroanatômicas encontradas. Gardner (1968), cria a teoria hidrodinâmica, em que a não perfuração da fossa rombóide acarretaria uma distensão do tubo neural com posterior deslocamento das estruturas neuroanatômicas localizadas na fossa posterior. McLone (1992) hipotetiza que a origem da MCII tenha correlação a um processo de neurulação comprometida. Acrescenta ainda que a não manutenção de um sistema ventricular com pressão adequada levaria a formação de uma fossa posterior com dimensões menores (SALMAN, 2011).

\section{Manifestações clínicas e achados no exame físico}

As manifestações neurológicas podem estar presentes após o nascimento (GRIEBEL et al., 1991). Nessa faixa etária, predomina o comprometimento dos nervos cranianos caudais que pode levar a um quadro de disfagia, estridor laríngeo, disfonia, paralisia musculatura cervical e opistótono (GRIEBEL, 1991; MCLONE, 1992). 
Após 12 meses de idade, os achados neurológicos mais comumente encontrados são déficits cerebelares, paresia em membros superiores e aumento do tônus. Raramente os pares cranianos são comprometido. (MCLONE, 1992).

Anormalidades intrínsecas do tronco encefálico (TE) juntamente com uma elevada pressão exercida sobre ele por causa de uma fossa posterior reduzida e hidrocefalia causam diversos achados como cefaleia, apneia, bradicardia, disfagia, torcicolo e espasticidade. Realizar a descompressão é necessária em $8 \%$ a 17\% dos pacientes e geralmente leva a uma melhora clínica. Déficits motores de fala, disfunção cognitiva e déficits de motricidade foram relatados em crianças com MMC.

Anormalidades do movimento ocular ocorrem na MCII, incluindo perseguição suave sacádica, dismetria sacádica, anormalidades do reflexo vestíbulo-ocular, nistagmo, estrabismo e oftalmoplegia internuclear. 0 registro do movimento ocular revelou que os pacientes com MCll com funções motoras oculares anormais tinham áreas menores da fossa posterior sagital mediana e seus volumes cerebelares eram menores do que os pacientes com Chiari Il que tinham movimentos oculares normais; enquanto a expansão sagital e o volume cerebelar mediano preservado corresponderam à preservação de algumas anormalidades de movimento ocular, apesar da redução geral no tamanho do cerebelo em MCII (SALMAN, 2011).

\section{Diagnóstico}

O diagnóstico radiológico da MCll pode ser realizado intra-útero, por meio de ultrassonografia ou dosagem de alfa-fetoproteína (pouco utilizado), ou após o nascimento através de tomografia computadorizada e ressonância magnética (OSBORN et al., 2014; MAIXNER W et al., 2008).

\section{Exame laboratorial}

A dosagem materna de alfa-fetoproteína é realizada no início do segundo trimestre gestacional, podendo ser determinado entre 14 e 21 semanas de gestação através da triagem para defeitos de fechamento de tubo neural. Atualmente esse exame está em desuso devido às várias limitações de sua dosagem laboratorial visto que níveis elevados de alfa-fetoproteína estão presentes em inúmeras condições clínicas fetais e também pelo transtorno gerado pela obtenção de um falso-positivo (BIZZI JWJ e MACHADO A, 2012).

\section{Ultrassonografia}

Em relação à triagem neonatal, a sensibilidade da ecografia fetal de alta resolução é próxima de $100 \%$ na detecção de defeitos de fechamentos do tubo neural (BIZZI JWJ e MACHADO A, 2012). A primeira ecografia é realizada no primeiro trimestre, entre 11 e 13 semanas de gestação. Embora não seja específico na constatação de malformações do tubo neural, este exame pode identificar anormalidades anatômicas fetais que podem corresponder a um risco aumentado para malformação fetal (BIZZI JWJ e MACHADO A, 2012).

Já ecografia obstétrica morfológica de segundo trimestre gestacional tem como objetivo estudar a anatomia interna e externa do feto para certificação de malformações ou defeitos congênitos. Idealmente deve ser realizada entre 20 e 22 semanas. As alterações mais encontradas associam-se muito com o desenvolvimento de mielomeningocele, que está intrinsecamente relacionada com a MCII (MAIXNER W, et al., 2008; BIZZI JWJ e MACHADO A, 2012). O primeiro sinal mais sugestivo é o "sinal do limão" que consiste em um cavalgamento ósseo frontal em decorrência de uma baixa pressão intracraniana fetal secundária a perda liquórica espinhal (MAIXNER W et al., 2008; BIZZI JWJ e MACHADO A, 2012).

A segunda alteração denomina-se "sinal da banana", que consiste em uma deformidade do tronco encefálico com um cerebelo alongado em formato bicôncavo envolvendo o tronco e obliterando a cisterna magna (BIZZI JWJ e MACHADO A, 2012).

\section{Ressonância magnética}

A ressonância magnética $(\mathrm{RM})$ fetal tem sido cada vez mais utilizada como exame de imagem não invasivo e completar à ultrassonografia (MAIXNER W et al., 2008; BIZZI JWJ e MACHADO A, 2012). Esse exame pode fornecer dados sobre o nível e extensão do defeito da coluna vertebral, ventriculomegalia e hemisférios displásicos. 


\section{Alterações anatômicas encontradas na MClI \\ Ossos do crânio}

O crânio lacunar que corresponde a um afilamento focal da calota craniana e aparência "escavada". A calvária aparece afilada com inúmeros defeitos circulares ou ovais e depressões rasas. Outro sinal evidente é a fossa posterior rasa e pequena com seio transverso em baixa posição, associado a um forame magno grande e "aberto" (OSBORN AG et al., 2014; MAIXNER W et al. 2008). Fossa posterior com volume menor com clivo e pirâmides petrosas côncavas quase sempre estão presentes.

\section{Dura-máter e tentório}

Anormalidades durais são muito comuns, como a incisura tentorial em forma de coração, grande e "aberta" e uma foice fina, hipoplásica e fenestrada, que permite que os giros cruzem a linha média. Em estudos de imagem a associação de interdigitações girais e foice deficiente resultam em uma aparência de fissura interhemisférica irregular (OSBORN AG et al., 2014; MAIXNER W et al. 2008).

Caso ocorra um deslocamento céfalo-caudal exagerado dos conteúdos da fossa posterior, os hemisférios cerebelares geralmente curvam-se anteromedialmente ao redor do tronco encefálico. Sinal do "cerebelo alto" pode ser encontrado na RM, quando o cerebelo e verme cerebelar são empurrados superiormente através da incisura (OSBORN AG et al.2014; MAIXNER W et al. 2008).

\section{Ventrículos cerebrais}

As anormalidades ventriculares também são reconhecidas frequentemente nos exames de imagem. Estão presentes em mais de $90 \%$ dos pacientes com MCII. Foto 1C. O IV ventrículo é deslocado caudalmente, alongado (quarto ventrículo em "canudo de refrigerante"). Foto 1D. O III ventrículo geralmente é grande e tem aderência intertalâmica bastante proeminente (OSBORN AG et al. 2014; MAIXNER W et al. 2008; GEERDINK $\mathrm{N}$, et al., 2018). Há também uma importante relação da MCll com hidrocefalia vistas concomitantemente em mais de $90 \%$ dos casos (GEERDINK N, et al., 2018). Quanto aos ventrículos laterais nota-se um apontamento inferomedial de cornos frontais, átrios colpocéfalos; ausência parcial de corpo caloso e septo pelúcido; fissura inter-hemisférica proeminente (MAIXNER W et al. 2008).

\section{Giros cerebrais}

Em relação aos hemisférios cerebrais, malformações do desenvolvimento cortical apresentam-se como polimicrogiria, heterotopia da substância cinzenta. Disgenesia do corpo caloso é encontrado em quase dois terços de todos os pacientes (OSBORN AG et al.,2014; MAIXNER W et al., 2008).

Podem ocorrer agenesia parcial ou completa do corpo caloso com ausência de septo pelúcido, comissura anterior proeminente, fissura longitudinal obliterada entre os lobos parietal e occipital e giro cingulado (GEERDINK N, et al., 2018). Alterações de estruturas límbicas também estão presentes, com hipocampos frequentemente pequenos e verticalizados. O lóbulo límbico posterior, incluindo giros hipocampal posterior e cíngulo posterior são desorganizados (MAIXNER W et al., 2008).

\section{Cerebelo e tronco encefálico}

As imagens de ressonância magnética nas ponderações T1 e T2 podem demonstrar o bulbo e o verme cerebelar deslocados inferiormente. Em cortes sagitais observa-se o deslocamento inferior do verme, bulbo e plexo coroide "em cascata" que se projeta inferiormente através do forame magno, situado atrás da medula espinhal. Outro sinal é o "teto em bico", formado quando o cerebelo herniado superiormente pode comprimir e deformar a placa quadrigeminal (OSBORN AG et al., 2014; GEERDINK N, et al., 2018)

Malformações mesencefálicas também podem ser demonstradas por exames de imagem, que demonstram ponto inferior da placa tectal com fusão do colículo inferior. Frequentemente o mesencéfalo encontra-se alongado ântero-posteriomente e transversalmente estreitado, presumivelmente por causa da presença do hemisfério cerebelar circundantes. $O$ aqueduto pode estar ocluso ou estreitado, o que pode ser considerado uma das possíveis causas de hidrocefalia (MAIXNER W et al., 2008). 


\section{Diagnóstico diferencial}

Os principais diagnósticos diferenciais de MCII são as demais malformações de Chiari (I, III e IV). A Malformação de Chiari I cursa com o deslocamento caudal das amígdalas cerebelares através do forame magno. Nela, mielomeningocele é ausente e a fossa posterior é relativamente normal, exceto pela dimensão reduzida. Em relação à Malformação de Chiari III, soma-se aos achados da MCII uma encefalocele occipital baixa ou cervical alta. No tipo IV, há hiperplasia cerebelar sem herniação (OSBORN et al., 2014). Lipomielomenningocele, meningocele "simples" e mielocistocele nunca são associadas a MC II.

\section{Tratamento cirúrgico}

\section{Derivação ventrículo-peritoneal}

A derivação liquórica associada ao controle da pressão intracraniana é de extrema importância, pois a hidrocefalia acompanha a mielomeningocele em aproximadamente $80 \%$ dos casos, e constitui fator extremamente adverso. No caso das alterações da dinâmica liquórica, ocorre aumento progressivo dos ventrículos cerebrais, levando à hipertensão intracraniana e macrocrania, com sofrimento cerebral acentuado. Essa complicação requer a necessidade de procedimentos cirúrgicos para derivação do líquor. Isso é feito através da instalação de sistemas para derivação liquórica. O mais comumente utilizado é o sistema de derivação ventrículo-peritoneal (ventriculoperitoneostomia) com interposição de uma válvula que regula a quantidade de líquor a ser drenado em função da pressão no interior do crânio. (PEREIRA et al., 2007).

\section{Craniectomia occipital e laminectomia cervical}

A descompressão da MCII pode ser desafiadora e requer entendimento completo da anatomia anormal a ser encontrada. Importante afastar a hidrocefalia como causa dos sintomas apresentados pelo paciente (STEVENSON, 2004). Em paciente com derivação ventrículo peritoneal é necessário também excluir que os sintomas decorrem de uma disfunção desta. Descartadas essas duas condições e se presente aumento de pressão intracraniana, realiza-se a descompressão através de laminectomia de C-1 (STEVENSON, 2004).

Daqueles pacientes portadores de malformação de Chiari sintomática, apenas $17 \%$ necessitarão de tratamento neurocirúrgico descompressiva da fossa posterior (STEVENSON, 2004). A descompressão cirúrgica da fossa posterior de pacientes com malformação de Chiari tipo II sintomática possui particularidades. Para programação cirúrgica é necessária uma RM da junção crânio-cervical, que orientará a extensão das laminectomias. Atenção para a posição da confluência dos seios venosos (tórcula) que possui implantação anormalmente baixa, muito próxima do forame magno. Em virtude disso e pela presença de seios venosos durais proeminentes nesses pacientes, o risco de hemorragia associado a uma abertura dural é grande necessitando muita cautela do neurocirurgião. Deve ser feita a plástica dural e não deve ser tentada a remoção do tecido neural do canal medular. Os resultados cirúrgicos se relacionam com a brevidade do tratamento, sendo relatado desaparecimento dos sintomas em $69 \%$ dos pacientes nos primeiros dois dias após o procedimento. A resolução imediata dos sintomas é o objetivo da descompressão cirúrgica e os relatos na literatura suportam uma abordagem cirúrgica agressiva ao MC II sintomático (STEVENSON, 2004).

A resolução dos sintomas pode ocorrer após algumas semanas. Além dos riscos imediatos de perda de sangue, infecção, lesão vascular e do sistema nervoso central, e persistência dos sintomas, o paciente com MCll enfrenta outros riscos de maneira mais tardia. A recorrência de sintomas após a descompressão do MC Il pode ser causada por mau funcionamento da derivação, descompressão inicial inadequada, cicatrizes epidurais com compressão em forma de faixa, e formação de espondilolistese cervical. Fatores que se mostraram associados à necessidade de eventual reoperação incluem a idade jovem na descompressão inicial e a falta de suprimento adequado do líquor (LCR) no quarto ventrículo. A instabilidade cervical e a cifose são complicações tardias bem conhecidas da laminectomia cervical em crianças (HANKINSON et al., 2011).

A descompressão da fossa posterior parece estar associada à normalização a longo prazo dos espaços cerebelares do LCR e a um fluxo de LCR presumivelmente melhorado dentro da fossa posterior. No entanto, este procedimento cirúrgico não oferece benefícios óbvios a longo prazo para o cerebelo, porque a atrofia 
cerebelar ainda era evidente nos pacientes com malformação de Chiari tipo II vários anos após o procedimento cirúrgico e era semelhante à atrofia cerebelar vista em pacientes com malformação de Chiari tipo II sem cirurgia descompressão (DENNIS M et al., 2004).

\section{Correção da mielomeningocele com técnica intra-uterina}

A correção neurocirúrgica da mielomeningocele intra-uterina tem sido uma opção disponível dentre os tratamentos atuais. Já existe algum grau de evidência científica de que essa técnica cirúrgica reduz a necessidade de derivação ventrículo-peritoneal, melhora a função cognitiva desses pacientes, bem como, eleva as chances de deambulação. (BEVILACQUA NS, PEDREIRA DAL, 2015).

\section{Correção da mielomeningocele com técnica intra-uterina via endoscópica}

A técnica neuroendoscópica é uma modalidade utilizada para o tratamento da MCII, entretanto, com insucesso inicial foi substituída pela técnica a céu-aberto. (DENNIS M et l., 2004). Trata-se de uma técnica que necessita de mais estudos ainda para avaliar à morbidade materna e fetal. (BEVILACQUA NS, PEDREIRA DAL, 2015).

\section{Prognóstico}

Apesar do crescente reconhecimento de MCII sintomática como uma anormalidade potencialmente fatal em crianças com mielomeningocele, até $15 \%$ dos pacientes com CM II morrem aos 3 anos de idade e quase um terço fica com incapacidade neurológica permanente (HANKINSON TC et al., 2011). As taxas de mortalidade relatadas nos pacientes com sintomas de disfunção de tronco cerebral após a descompressão cirúrgica foram de 50 a 70\% antes de uma abordagem cirúrgica mais agressiva. A elevada taxa de mortalidade foi atribuída ao dismorfismo de tronco cerebral intrínseco e / ou isquemia e hemorragia do tronco encefálico. (HANKINSON TC et al., 2011).

\section{CONSIDERAÇÕES FINAIS}

As malformações de Chiari Il estão inseridas no espectro de anormalidades congênitas do sistema nervoso central. Os pacientes apresentam a mielomeningocele e hidrocefalia na maioria dos casos. Essas alterações anatômicas repercutem negativamente no desenvolvimento cerebral fisiológico o que resulta em elevada morbimortalidade dos portadores dessa síndrome. $\mathrm{O}$ tratamento definitivo abrange diferentes modalidades cirúrgicas tais como derivação ventrículo-peritoneal, descompressão da fossa posterior, além do reparo da mielomeningocele intra-útero.

\section{REFERÊNCIAS}

1. BEVILACQUA NS, PEDREIRA DAL. Cirurgia fetal endoscópica para correção de mielomeningocele: passado, presente e futuro. Einstein, 2015; 13 (2):283-9.

2. BIZZI JWJ, MACHADO A. Mielomeningocele: conceitos básicos e avanços recentes. Jornal Brasileiro de Neurocirurgia, 2012; 23 (2):138-151.

3. CAI C, OAKES WJ. Hindbrain herniation syndromes: The Chiari malformations (I and II). Sem Pediatr Neurol, 1997; 4:179-91.

4. SILVA JA. Basilar impression and Arnold-Chiari malformation surgical findings in 209 cases. Neurochirurgia (Stuttg), 1992; 35 (6):189-95.

5. DENNIS M, et al. Neurobiology of perceptual and motor timing in children with spinabífida in relation to cerebellar volume. Brain, 2004; 127(6): 1292-1301.

6. GARDNER WJ. Myelocele: rupture of the neural tube? Clin Neurosurg, 1968;15:57-79.

7. GEERDINK N, et al. Essential features of Chiari II malformation in MR imaging: an inter observer reliability studypart 1. Child's Nervous System, 2012; 28 (7): 977-985.

8. GRIEBEL ML, et al. The Chiari malformation associated with mielomeningocele. In Rekate HL (ed). Comprehensive management of spina bifida. Boca Raton: CRC Press,1991:67-92.

9. HANKINSON TC, et al. Myelomeningocele and Myelocystocele in Youmans Neurological Surgery. EUA: Editora Saunders Elsevier, 2011. Volume 2. 
10. MAIXNER W, et al. The Spina Bifida: Management and outcome. $1^{\text {st }}$ ed. Springer, 2008; 532p.

11. MCLONE DG. Continuing concepts in management of spina bifida. Pediatr Neurosurg, 1992;18:254-256.

12. MCLONE DG. Results of treatment of children born with a myelomeningocele. Clin Neurosurg, 1983; 30:407-435.

13. OSBORN AG, et al. Malformações da fossa posterior. In: Osborn's brain imaging pathology and anatomy. Porto Alegre: Artmed Editora Ltda, 2014; Cap. 36. p. 1065-1091.

14. PENFIELD W, COBURN DF. Arnold-Chiari malformation and its operative treatment. Arch Neurol Psychiat, 1938; 40:328-336.

15. PEREIRA ELR, et al. Epidemiologia de pacientes com malformação de Chiari II internados no Hospital Fundação Santa Casa da Misericórdia do Pará. Rev. Para. Med, 2007; 21(2).

16. SALMAN MS. Posterior fossa decompression and the cerebellum in Chiari type II malformation: a preliminary MRI study. Child's Nervous System, 2011; 27 (3): 457-462.

17. SBRAGIA L, et al. Evolução de 58 fetos com meningomielocele e o potencial de reparo intra-útero. Neuropsiquiatria, 2004; 62(2-B).

18. STEVENSON KL. Chiari Type II malformation: past, present, and future. Neurosurg Focus, 2004; $16(2)$ : E5.

19. VENES JL. Multiple cranial nerve palsies in an infant with Arnold-Chiari malformation. Dev Med Child Neurol, 1974;16:817-820.

20. WATERS KA, et al. Sleep-disordered breathing in children with myelomeningocele. J Pediatr, 1998;132(4):672-81. 